\title{
Fuzzy Logic Based Flood Warning and Floodgate Control System
}

\author{
Yogesh Waghmare, Prakash Choudhary, Mohit Rochlani and Manisha Rajput
}

\begin{abstract}
This paper is about the construction of flood warning system using the embedded controller. Floods have large social consequences for communities and individuals. To tackle all these issues, the proposed mechanism constructs flood warning system using the embedded controller Atmega 32. Here the main aspect is to control the gate of the main reservoir using the fuzzy logic in a precise manner. Controlling of the gate is based on the different inputs from the sensors situated at small reservoirs. So that there will be fewer chances of flood and it saves the dam from the maximum damage, and area nearby dam will also be safe from the flood The conventional control techniques is replaced here by using fuzzy logic and implemented using low-cost Atmega32 microcontroller to provides a solution.
\end{abstract}

\section{Keywords}

Control System

Embedded system;

Floodgate Control System;

Fuzzy logic controller;

Flood warning system;

Ultrasonic sensors.

\section{INTRODUCTION}

The immediate impacts of flooding include loss of human life, damage and destroy property, loss of livestock, destruction of crops, and worsening of health conditions owing to waterborne diseases [1]. The construction of a dam is necessary for flood control, irrigation system, and metropolitan, the electric power generation, and industrial water supply [2]. The modern day technologies in the field of information storage and retrieval like web search, image processing, pattern recognition, bioinformation and computational biology, emarkets, autonomous navigation, and guidance are all benefited greatly using something called Fuzzy Sets [3]. The conventional flood control techniques [4-8] is replaced here by using fuzzy logic and implemented using low cost [9] Atmega32 microcontroller to provides a solution. This proposed work is basically used to control the dam reservoir gate through fuzzy logic implemented on embedded system using ultrasonic sensors and the gate is controlled in a precise manner by the steps of stepper motor and the problem of flooding due to excess water in the reservoir will be solved. Previously the gate control is done manually [10], if the amount of water increases above the certain level then gate will shift by some level and water will be released, but when suddenly water level increases then there will be chances of overflowing the reservoir and area near too that reservoir is also affected by the flood, so it takes thousands of life and millions of property loss. The conventional control techniques is replaced here by using fuzzy logic and implemented using low cost Atmega32 microcontroller to provides a solution.

\section{PROPOSED SYSTEM}

This section is about construction of flood warning system using the embedded controller. The programming of the Atmega32 microcontroller is done in $\mathrm{C}$ language by using software 'Mikro C Pro for AVR' [11]. To control the gate of the main reservoir fuzzy algorithm is used. Here the demo shows some small reservoirs in the way from where large amount of water is coming in the main dam, and controller is responsible for the flow and amount of the water. This paper proposed a mechanism to control the dam's gate via the embedded controller. System is introducing some small reservoirs which will be far distance from main reservoir. In these reservoirs ultrasonic sensors are situated which will regularly calibrating the water level and are interfaced to the fuzzy controller installed in the main reservoir. Analog signals as three different inputs are given to the controller. As a consequence, microcontroller will decide the opening and closing of the gate based on those analog inputs. The shifting of gate depends on the instructions given to the microcontroller. Here the fuzzy logic is used as an algorithm to perform tasks given by rules to the controller. All values are calibrated by sensors and are shown on LCD display step by step to operate the gate. Based on those inputs stepper motor will rotate by different steps depending on the in fuzzy logic rules set in the microcontroller. Here basically 
angular moment of motor results into linear moment with the help of rack and pinion arrangement to drive the pulleys. The floodgates are control using motor controller unit (Fig 1). A remote operator can access real time data from desktop.

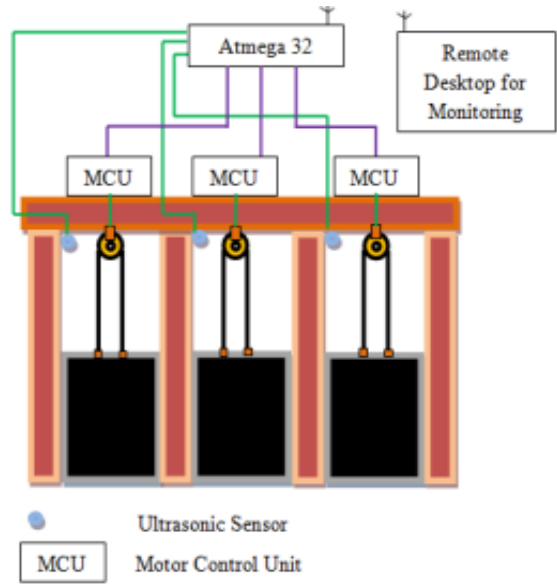

Fig 1. Illustration of the proposed system.

\section{Fuzzy Sets And Acoustic Principle}

Rule base defined how microcontroller will decide output depending upon the various inputs. For that various threshold values is defined in the rule base. Based on statistic fuzzy sets are posed as Table1. Depending on the output of the sensor unit in terms of water level buzzer as well as motor take necessary action and send these data to the remote controller. Ultrasonic waves works same as sound waves, they can travel through medium. So they are used in proximity and distance learning applications. Ultrasonic transducer transmits a short burst of sound waves towards the target and it reflects back to the sensors, by which distance is calculated using return of the echo pulses. The frequency of the ultrasound wave is normally $40 \mathrm{kHz}$ to $250 \mathrm{kHz}$.Due to large frequency range it vary according to different material. Some of the principles are given below [12-13],

(1)Variation in speed as a function of both temperature and composition of the transmission media.

(2) Variation in wavelength vary with function of speed and frequency.

(3) Variation in attenuation with function of frequency and humidity.

(4) Variation in amplitude of return echo pulse vary with target distance, geometry, size and surface.

(5) $\mathrm{I}=\mathrm{I} 0 * \mathrm{e}-\alpha \mathrm{x}$ where I and I0 are the received and original intensities respectively. ' $\alpha$ ' is attenuation coefficient, and ' $\mathrm{x}$ ' is distance travelled by the wave.
The speed of sound waves in that medium or fluid is given by the Eq (1).

$$
c=\sqrt{\frac{k}{p}}
$$

where $\mathrm{k}=$ bulk modulus $\mathrm{p}=$ density. Using this parameter we fetch the information from sensors. The "then" part is called the rule consequent and is description of the control output. Here, a fuzzy control rules provide a convenient way for expressing control policy and domain knowledge. The choice of process state variables and control variables is compulsory for the characterisation of the operation of a fuzzy system.

Table 1. Proposed Fuzzy rule

\begin{tabular}{|c|c|c|c|c|}
\hline $\begin{array}{l}\text { Sr. } \\
\text { no }\end{array}$ & $\begin{array}{c}\text { Sensor } 1 \\
(\mathrm{ft})\end{array}$ & $\begin{array}{c}\text { Sensor } 2 \\
(\mathrm{ft})\end{array}$ & $\begin{array}{c}\text { Sensor } 3 \\
(\mathrm{ft})\end{array}$ & $\begin{array}{c}\text { Result } \\
\text { Buzzer(B), Motor(M) }\end{array}$ \\
\hline 1 & $0<x<10$ & $0<x<10$ & $0<x<10$ & No action \\
\hline 2 & $10<x<15$ & $0<x<10$ & $0<x<10$ & B $2 \mathrm{sec}$ \\
\hline 3 & $0<x<10$ & $0<x<10$ & $10<x<15$ & B 2sec \\
\hline 4 & $0<x<10$ & $10<x<15$ & $0<x<10$ & B 2sec \\
\hline 5 & $15<x<20$ & $0<x<10$ & $0<x<10$ & B 4sec, M 4 unit \\
\hline 6 & $0<x<10$ & $15<x<20$ & $0<x<10$ & B 4sec, M 4 unit \\
\hline 7 & $0<x<10$ & $0<x<10$ & $15<x<20$ & B 4sec, M 4 unit \\
\hline 8 & $20<x<30$ & $0<x<10$ & $0<x<10$ & B 4sec, M 6 unit \\
\hline 9 & $0<x<10$ & $20<x<30$ & $0<x<0$ & B 4sec, M 6 unit \\
\hline 10 & $0<x<10$ & $0<x<10$ & $20<x<30$ & B 4sec, M 6 unit \\
\hline 11 & $10<x<15$ & $0<x<10$ & $10<x<20$ & B 2sec, M 2 unit \\
\hline 12 & $15<x<20$ & $10<x<20$ & $0<x<10$ & B 2sec, M 4 unit \\
\hline 13 & $15<x<20$ & $10<x<20$ & $15<x<20$ & B 4sec, M 6 unit \\
\hline 14 & $20<x<25$ & $20<x<30$ & $15<x<30$ & B 4sec, M 8 unit \\
\hline 15 & $10<x<20$ & $10<x<15$ & $0<x<10$ & B 2sec, M 2 unit \\
\hline 16 & $0<x<10$ & $15<x<20$ & $10<x<20$ & B 2sec, M 4 unit \\
\hline 17 & $15<x<20$ & $15<x<20$ & $10<x<20$ & B 4sec, M 6 unit \\
\hline 18 & $15<x<30$ & $20<x<25$ & $20<x<30$ & B 4sec, M 8 unit \\
\hline 19 & $0<x<10$ & $10<x<15$ & $10<x<15$ & B 2sec, M 2 unit \\
\hline 20 & $10<x<15$ & $15<x<20$ & $15<x<20$ & B 2sec, M 4 unit \\
\hline 21 & $10<x<20$ & $20<x<25$ & $15<x<20$ & B 4sec, M 6 unit \\
\hline 22 & $20<x<30$ & $20<x<25$ & $20<x<25$ & B 4sec, M 8 unit \\
\hline
\end{tabular}

\section{ReSUlt ANALYSIS}

By this mechanism controlling the dam's gate is possible through embedded system. output of embedded system depends upon the specific rules and rules will driven by the different inputs. Matlab simulation result of membership function for all input functions are shown in Fig 2. Depending upon various inputs motor will rotate both in clockwise

Yogesh Waghmare, Prakash Choudhary, Mohit Rochlani and Manisha Rajput, "Fuzzy Logic Based Flood Warning and Floodgate Control System," International Journal of Advanced Engineering and Management, Vol. 2, No. 7 , 


\section{JOAEM}

and anticlockwise direction. if water level increases then motor will rotate to lift the gate and vice versa. The rule viewer shows how the output change with respect to the input and the surface viewer shows the $3 \mathrm{~d}$ view of the output with respect to inputs (Fig 3).

For an example, input (1): 5, input (2): 12 input(3): 21 motor will rotate in anti clock wise direction 4 unit. If this water level changes and decreases by some level, For an example, input (1):12, input(2):-8, input(3):-9 motor will rotate in clock wise direction.

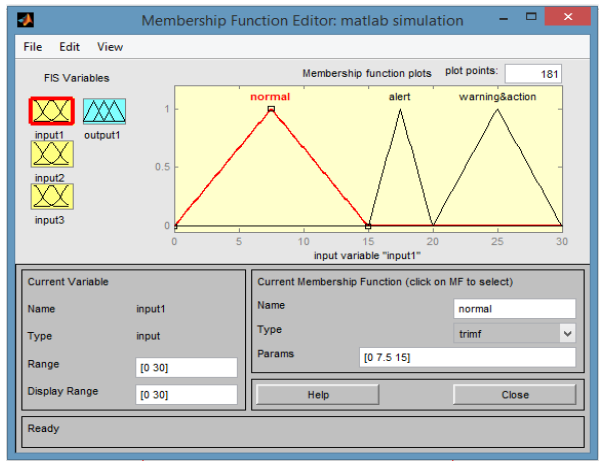

(a)

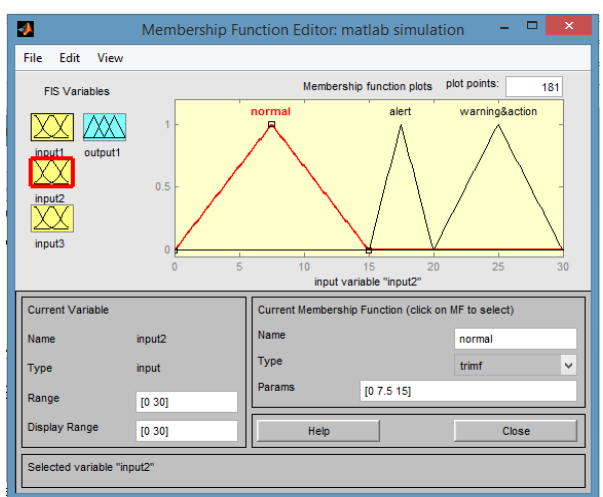

(b)

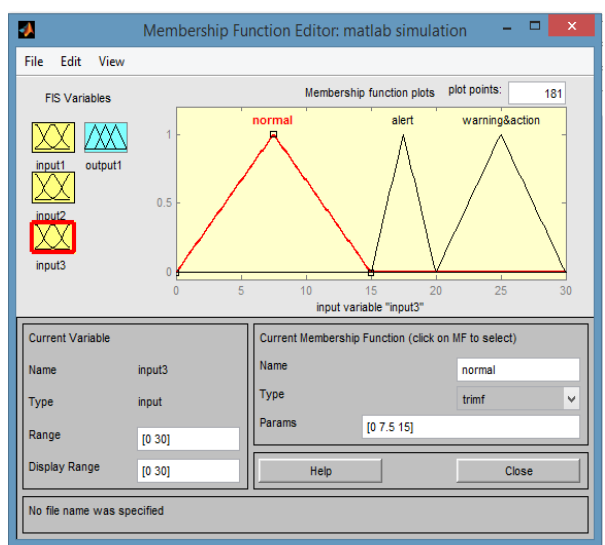

(c)

Fig 2. Matlab simulation of membership function for (a) input 1, (b) input 2 and (c) input 3.

Yogesh Waghmare, Prakash Choudhary, Mohit Rochlani and Manisha Rajput, "Fuzzy Logic Based Flood Warning and Floodgate Control System," International Journal of Advanced Engineering and Management, Vol. 2, No. 7, pp. 172-175, 2017. DOI: https://doi.org/10.24999/IJOAEM/02070039

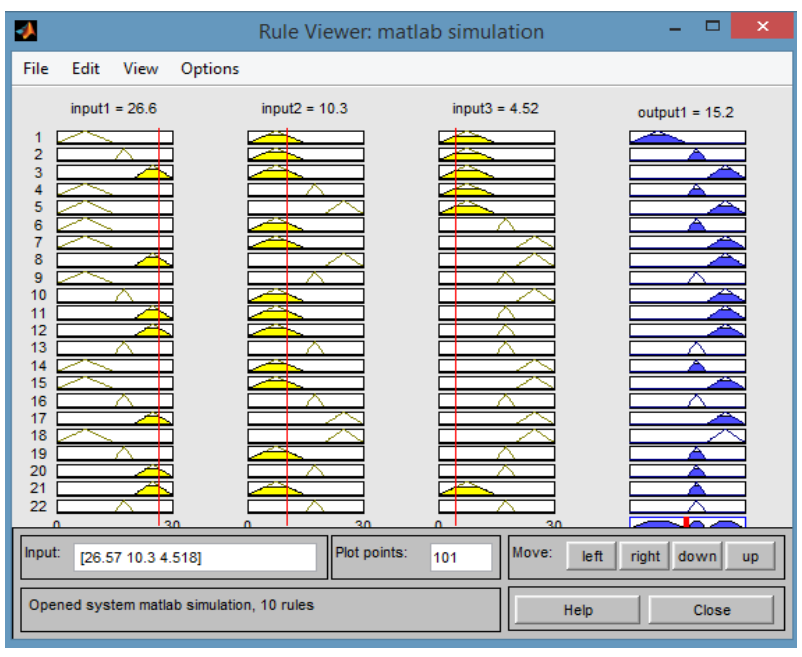

(a)

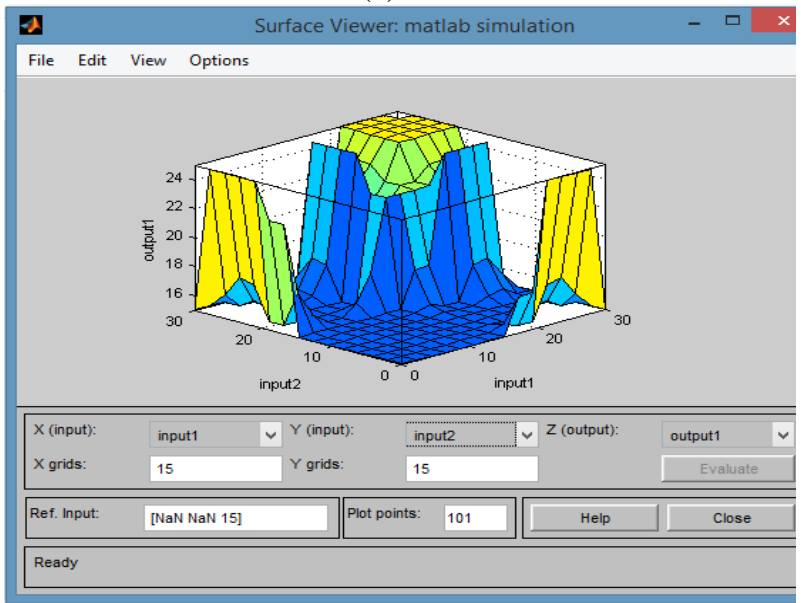

(b)

Fig 3. (a) This rule viewer shows how the output changes with respect to the input and $(b)$ surface viewer shows the $3 d$ view of the output with respect to inputs.

This is done for not to empty the dam, some water should remain in dam for the future uses so anticlockwise movement is also compulsory. So according to the level gate of the dam is controlled precisely by implementing this system in practise. With this mechanism we can save thousands of people life who live near the dam. Proposed mechanism is capable of handling most of the problem due to which failure of the dam take place. also mechanism will able to reduce man power and less supervision required.

The controller so designed can be applied to a many fields. During the course of investigation of the present work, it has been found that there is a scope for future work in the following area. In the present work vast expert decision maker, in home automation, automatic break control in opening of door of heavy gate. The following figures are the 


\section{ESTD. 2016}

simulation of the Matlab. The inputs are decided by the different membership functions which results into the crisp value output. Rules are defined and their variation can be seen in rule viewer. In rule viewer how the output changes with respect to the input is verified.

\section{Conclusion}

The application of fuzzy logic controller it is tried to obtain a constant output voltage for a given input reference voltages by which a warning system is designed. This mechanism of opening and closing of gate is possible automatically by the embedded system. Till this time control of floodgate done manually, failure of the dam take place. Due to small reservoir we can predict the amount of water coming to the main reservoir, if large amount of water certainly come from small reservoir then small amount of water continuously releases from the main reservoir. Because of this the main reservoir will never fill completely and the condition of flood will not be there.

\section{REFERENCES}

[1] Kozlowski, T. T. (2002). Physiologicalecological impacts of flooding on riparian forest ecosystems. Wetlands, 22(3), 550-561.

[2] Le, T. V. H., Nguyen, H. N., Wolanski, E., Tran, T. C., \& Haruyama, S. (2007). The combined impact on the flooding in Vietnam's Mekong River delta of local man-made structures, sea level rise, and dams upstream in the river catchment. Estuarine, Coastal and Shelf Science, 71(1), 110-116.

[3] Otto, M. (1988). Fuzzy theory explained. Chemometrics and intelligent laboratory systems, 4(2), 101-120.

[4] Rinkewich, I. (1995). U.S. Patent No. 5,427,350. Washington, DC: U.S. Patent and Trademark Office.

[5] Changyi, L. (2005). The Design of Metro Flood Gate [J]. Urban Rapin Rail Transit, 4, 032.

[6] Wu, C. L., \& Chau, K. W. (2006). Mathematical model of water quality rehabilitation with rainwater utilisation: a case study at Haigang. International Journal of Environment and Pollution, 28(3-4), 534- 545.

[7] Ding, Y., \& Wang, S. S. (2012). Optimal control of flood diversion in watershed using nonlinear optimization. Advances in Water Resources, 44, 30- 48.

[8] Guan, H. L., Chi, Y. L., Dai, H. Z., \& Yang, Y. (2008). Small signal stability and control of wind turbine with asynchronous generator integration into power system. Automation of Electric Power Systems, 32(4), 54-58.

[9] Kumar, M., Kabir, F., \& Roy, S. (2017). Low Cost Smart Stick for Blind and Partially Sighted People. International Journal of Advanced Engineering and Management, 2(3), 65-68.

[10] Purnaningrum, E., \& Apriliani, E. (2016). Auto Floodgate Control Using EnKf-NMPC Method. International Journal of Computing Science and Applied Mathematics, 2(1), 14.

[11] Asif, O., Hossain, M. B., Hasan, M., Rahman, M. T., \& Chowdhury, M. E. (2014). FireDetectors Review and Design of an Automated, Quick Responsive FireAlarm System Based on SMS. Int'l J. of Communications, Network and System Sciences, 7(09), 386.

[12] Schmid, F., Grbin, P., Yap, A., \& Jiranek, V. (2011). Relative efficacy of high pressure hot water and high power ultrasonics for wine oak barrel sanitization. American journal of enology and viticulture, ajev-2011.

[13] Falkenström, M., Engman, M., LindhUlmgren, E., \& Hutchinson, B. (2011). Laser ultrasonics for process control in the metal industry. Nondestructive Testing and Evaluation, 26(3-4), 237-252.

\section{Yogesh Waghmare}

yogeshwaghmare118@gmail.com

Department of Electronics and Telecommunication, Dr. D. Y. Patil College of Engineering, Pune, India Prakash Kumar

kumarprakash358@gmail.com

Department of Electronics and Telecommunication, Dr. D. Y. Patil College of Engineering, Pune, India Mohit Rochlani

mohitrochlani09@gmail.com

Department of Electronics and Telecommunication, Dr. D. Y. Patil College of Engineering, Pune, India Manisha Rajput

rajput_manisha@rediffmail.com

Department of Electronics and Telecommunication, Dr. D. Y. Patil College of Engineering, Pune, India Editor-in-Chief: Sahadev Roy 